\title{
ENABLING POSTGRADUATE LEARNING IN THE WORKPLACE
}

Nicola Beasley ${ }^{1}$, John Ford ${ }^{2}$, and Nils Tomes ${ }^{2}$

${ }^{1}$ Napier University, Edinburgh, Scotland; ${ }^{2}$ Heriot-Watt University, Edinburgh, Scotland

\begin{abstract}
This paper describes a research project that aimed to determine and evaluate the learning environment customisations required to support self-motivated, able, and experienced learners studying towards a Masters degree in Petroleum Engineering within small to medium-sized enterprises (SMEs). The requirements analysis of the specific needs of workplace learners, the system customisation and evaluation are described. The evaluation showed that organisational factors are a key factor in achievement, that students tended to study sequentially even in a hyperlinked environment, and that they valued a combination of online and printed materials.
\end{abstract}

Key words: distance learning, computer assisted instruction, higher education

\section{INTRODUCTION}

An online study environment can be an effective medium for selfmotivated students to study at work (Gray, 1999; Brink et al., 2002; Harun, 2002). This paper describes how a learning environment can be developed to support this type of independent learning. The EC-funded CBLPET (Computer-Based Learning in Petroleum Engineering) project, described here, builds on previous research in the development of constructivist learning environments (Grabinger \& Dunlap, 1995; Hannafin \& Land, 1997; Jonassen \& Murphy, 1999) and computer-based learning for skills-based training (Ferreira et al., 1998) to identify possible customisations for an online learning environment that could support Masters-level, workplacebased students of Petroleum Engineering, and to evaluate the effectiveness of that environment. 
The project aimed first to define the ways in which the learning requirements for postgraduate, workplace-based students differ from the requirements of lower-stage, campus-based students, and to design a learning environment taking this into account. The second aim was to evaluate the effectiveness of this learning environment.

In the sections that follow, the project is described in more detail; the process of eliciting the user requirements is explained; the evaluation methodology is specified; the evaluation results described; and finally, conclusions are drawn from the research.

\section{DESCRIPTION OF PROJECT}

The development of the CBLPET learning environment was primarily based on previous research in the design of online learning environments undertaken in the EU-sponsored ASTEP (Advanced Software for the Teaching and Evaluation of Processes) Project (MacKinnon et al., 1998). ASTEP differs from CBLPET in that it focuses on process-based technical training, so the first stage of this project was to perform an evaluation of which elements from the ASTEP framework were appropriate for the new learning environment, and which additional elements would need to be added to meet the specific needs of the degree, student group and industry.

The Masters degree in Petroleum Engineering is a one-year, intensive, highly demanding campus-based course, which is also available as a paperbased distance learning course, aimed at graduate engineers. The student population being studied were undertaking study while working in the Petroleum Engineering industry, came from a range of nationalities, and had a broad range of graduate-level scientific and technical knowledge.

The petroleum industry is international with a highly mobile workforce. Not only do students reside across the globe, they may be expected to move location at short notice; therefore an environment that allows continuity of study is of prime importance. Workers require up-to-date multidisciplinary skills to stay employable in a quickly changing market, and a significant proportion of the workforce are based in small to medium-sized enterprises, with heavy demands on their time, and where there are often no subject experts locally available. SMEs are also characterised by having small training budgets and limited release time for staff (Bradley \& Oliver, 2002) and these factors reinforce the efficacy of online learning.

The requirements analysis for this project was based on a series of interviews, questionnaires, and an industry analysis. This was followed by the production of a prototype online environment containing two of the MSc course modules in web format, which were used by students to study for the 
relevant examinations. The final stage of the project was to conduct an evaluation of the system to assess its effectiveness and draw out a set of recommendations for further research and development.

It was hypothesised that online learning would significantly enhance the availability, affordability and flexibility of advanced training while delivering the essential quality of learning needed at this level.

\section{ELICITATION OF REQUIREMENTS}

The purpose of the requirements analysis was to identify elements of the ASTEP environment that would be appropriate for the student population under study, and additions that could be made to the existing learning environment to make it more suitable for workplace-based MSc students.

The ASTEP framework is a task-centred model for development of learning environments, which includes thirteen recommendations for technical components, covering the areas of student management, assessment, student portfolios, communication, online identity, activities, materials and evaluation. As it is a model designed for teaching processes face-to-face, some components - portfolios, synchronous communication, process diagnosis activities - were not seen as appropriate for this project; however, the framework provided a good basis on which to begin.

The requirements analysis consisted of three stages: collection of data from students, mentors, academic, technical and managerial staff, using questionnaires and interviews; a market analysis of the small to mediumsized enterprise segment of the petroleum engineering market; and an evaluation of the ASTEP framework in relation to these results.

These analyses provided a number of requirements for workplace learners, many of which were commonly accepted constructivist learning environment components such as learning activities, setting learning within an authentic context, provision of information resources (Grabinger \& Dunlap, 1995; Jonassen \& Murphy, 1999) and are therefore not discussed further here. There follows a discussion of the key additional features implemented, which the analysis indicated would be of value in supporting mature, work-based learners in petroleum engineering.

Four key features to support these learners were identified in the requirements analysis. First, although communication systems are a basic feature of many constructivist learning environments, they are highlighted here for a number of reasons. Students on the course are geographically dispersed, working in small organisations with no subject specialist readily available, so it was considered that an effective way of communicating with academics, and other students on the course, was of prime importance. The 
use of a synchronous communication system was not thought to be appropriate, owing to international time differences, so this need was met using an asynchronous discussion forum.

Secondly, it was considered that a way of storing user data to personalise the system and store data regarding tasks completed was essential because students may not be working in fixed locations and would still need to see where they are in the materials and what work needs to be completed.

Thirdly, students come from diverse undergraduate backgrounds and it is common for many to experience difficulty with those modules on the course that contain new technical terminology and a large subject vocabulary, particularly students whose first language is not English. There was therefore a requirement for an easily accessible system for displaying terminology, which was implemented using an extensive hyperlinked glossary.

Finally, on the technical aspects of delivery, since students are based in locations worldwide, it is essential to use technology that is available to all. Also, students studying at work may not have exclusive access to a computer, and may not have permission to modify the set-up. For this reason plug-ins and use of machine customisations were kept to a minimum.

Students were supported on a day-to-day basis by company mentors and managers, as well as academic and technical staff at Heriot-Watt University.

The evaluation focused on those features which, on the basis of the discussion above, it was expected would add particular value to the target users, as well as examining the ways in which the environment was used by students, and identifying improvements for future iterations of the course.

\section{EVALUATION METHODOLOGY}

The aims of the evaluation were to identify which requirements were adequately met, and which needed further research and development; to discover how the learning environment was being used in an organisational setting; and to catalogue ideas and suggestions for future improvements. Two student groups took part in the evaluation of the prototype modules:

- Distance learning students enrolled on the course and using paper-based materials while studying at work were offered access to the online system and 18 students agreed to take part in the evaluation. They were asked to complete a questionnaire detailing their first impressions after three weeks (12 returns) and to complete a second, in-depth questionnaire after completing the course (4 returns). While the response rate from the second questionnaire was disappointing, the questionnaires that were completed were thorough and insightful. 
- Two small-to-medium-sized petroleum-engineering companies (in Scotland and Norway) were selected to take part in this study, and each company put forward two individuals who were interested in studying online. These individuals took part in an in-depth evaluation involving interviews and walkthroughs of common tasks within the system.

It is interesting to note that despite initial interest from 18 distancelearning students, only 12 actually accessed the system. Where reasons for this were given they tended to focus on lack of free time, and the fact that students were already used to working with the paper manuals.

In addition to the evaluations completed with students, interviews were carried out with company mentors, academic, managerial, and technical staff who had been using the system to support students.

\section{EVALUATION RESULTS}

The prototype system was received positively by the vast majority of students that used it; they appreciated its simple usable interface, ease of navigation and appropriate content.

Initially, students were provided only with access to the online materials but they asked to be provided with printed copies of the materials too. The students appreciated the flexibility of working without a computer that access to paper copies of materials afforded them. Although the students involved in the trial were allowed to study at work they were actively encouraged to work at home in their own time, and, in fact, most students worked predominantly with the paper copy at home, and with the computerbased materials at work. This preference for working on paper has also been found in similar studies (Crook, 1997; Ward \& Newlands, 1998). The evaluation showed that most students found that studying at work during working hours was difficult owing to constant noise, interruptions, and large amounts of time spent away from the desk, and most favoured working at home or in the office, out of hours. The inability to annotate the web-based material was also cited as a reason for preferring to work on paper.

A suggestion for improving the ability to study in the workplace would be the provision of approximate timings for topics and activities. Students noted that when studying in the workplace they often had definite time periods in which to study; it was therefore suggested that timed 'lessons' of different lengths would be very beneficial in enabling workplace study. There was a marked difference between the performances of the students studying at the Scottish company compared to those at the Norwegian one. The evaluation indicated that this was due to the increased sense of isolation felt by the Norwegian students. 
An asynchronous discussion forum was provided for students to ask subject-specific questions of the university-based academic staff, but was used by few of the students. The main reason was that they genuinely saw no need for such a feature as they could gain all the support they needed from the company mentor and preferred to get that support face-to-face, although some students were discouraged by the lack of activity on the forum.

A personalised reporting system was also provided for students to monitor their progress through the materials and identify which sections they had yet to finish, and this was also universally unused by the students as they felt they had no need for such functionality. The students tended to study in a linear fashion rather than by following hyperlinks so it was obvious to them which sections still had to be completed. However, academic staff found this feature useful for monitoring student work patterns and progress.

The use of a hyperlinked glossary system was found to be extremely useful by all students, although there were some technical issues with download times over slow connections. The ability to use a glossary term to look up its references in the accompanying text was cited by several students as a worthwhile future improvement.

The fact that simple technology was used was approved by most students, who appreciated the speed with which the site functioned. The students preferred a fast and functional system to a slow and fancy one. There were few technical issues associated with accessing and using the site.

An interesting finding was the way in which students used the learning activities embedded in the course, which consisted of self-test questions, worked examples, and reflective activities. The online system is designed in such a way that activities can be used to support learning in two ways - to consolidate learning after studying sections of the material, or to act as a structure in itself, using the activities to guide learning. Students indicated that they felt these activities were a core part of the course, but they were used exclusively in the former context. Learners preferred to study in a linear fashion, using the materials as a basis from which to access the activities. An interesting consideration for further study is whether this is due to a true preference for linear study, a preference for paper-based materials and a need to use the online materials in conjunction, or simply a lack of experience and support in non-linear forms of learning.

Interactive course elements, although identified in the requirements analysis, were not prioritised in the prototype system. However, a section of the evaluation focused on the areas of the course where appropriate interactions could best add value, and what those types of interactions should be. Suggestions that are particularly appropriate to the target group include:

- glossary word-matching quick quizzes to test knowledge in areas that depend on knowing a lot of terminology; 
- animated graphics of geological and time-dependent processes;

- the ability to isolate separate data segments on complex graphs;

- hints, such as bringing up a solution to a problem a line at a time, so that keen learners can pick up the problem from where they understand it.

\section{CONCLUSIONS}

The evaluation conducted in this study highlighted a number of findings for further research, and suggested several key improvements that would help to improve postgraduate workplace learning in petroleum engineering.

A main issue associated with workplace learning is that of balancing study and work time as well as dealing with noise and distractions. Managerial and mentor support is key to successful study at work. The restructuring of the course into timed lessons, which can be scheduled into the working day, would enable students to better plan their workloads.

Students worked with both paper-based and online materials showing a preference for paper-based outside the workplace, and it is evident that in the petroleum engineering industry a mixed model of home and workplace, paper-based and online delivery is required. It is apparent that online learning should not be seen as a replacement for the printed study guides currently used in the Masters course in Petroleum Engineering, but should be used strategically to complement and support them by providing appropriate communication facilities, hyperlinked glossaries, Internet links to information, interactivities, and online formative assessment. Students tended to work through the materials in a linear rather than hyperlinked fashion, using activities to support the materials and not vice versa.

Further investigation in this area would be useful to try to identify the reasons this might be so. Other potential areas of research include a study of the key factors that lead to isolation and how a learning environment could be used to counter them, and an investigation of ways in which to practically counteract the barriers to students using the online materials in the workplace. In all, this study has shown that an online learning environment in combination with paper-based resources - can be an effective medium for postgraduate workplace learning. However, issues such as student isolation, improved use of the potential of the web through increased interactivity and communication, and organisational support need to be addressed in future developments. 


\section{REFERENCES}

Bradley, C. \& Oliver, M. (2002). Developing e-learning courses for work-based learning. Paper presented at the Eleventh International World Wide Web Conference, Hawaii. [Available online] http://www2002.org/CDROM/alternate/703/

Brink, B., Munro., J. \& Osborne, M. (2002). Online Learning Technology in an SME WebBased Setting. Educational Technology and Society, 5(2), 81-85.

Crook, C. K. (1997). Making hypertext lecture notes more interactive: undergraduate reactions. Journal of Computer Assisted Learning, 13, 236-244.

Ferreira, M., MacKinnon, L., Desmulliez, M. \& Foulk, P. (1998). A multimedia telematics network for on-the-job training, tutoring and assessment. Proceedings of the International Conference on Engineering Education, Rio de Janeiro.

Gray, D. (1999). Work-based Learning, Action Learning and the Virtual Paradigm. Paper presented at the European Conference on Educational Research, Lahti, Finland.

Hannafin, M. J. \& Land, S. M. (1997). The foundations and assumptions of technologyenhanced student-centered learning environments. Instructional Science, 25, 167-202.

Harun, M. H. (2002). Integrating e-Learning into the workplace. The Internet and Higher Education, 4, 301-310.

Jonassen, D. H. \& Murphy, L. (1999). Activity Theory as a Framework for Designing Constructivist Learning Environments. Educational Technology, Research and Development, 47(1), 61-79.

Grabinger, R. S. \& Dunlap, J. C. (1995). Rich environments for active learning: a definition. ALT-J, 3(2), 5-34.

MacKinnon, L. M., McAndrew, P. \& Flockhart, S. (1998). ASTEP Framework User Manual. [Available online] http://www.cee.hw.ac.uk/ astep/deliverables/d24v12.pdf

Ward, M. \& Newlands, D. (1998). Use of the Web in undergraduate teaching. Computers \& Education, I, 171-184.

\section{BIOGRAPHY}

Nicola Beasley is an Academic Development Adviser at Napier University. She has worked in the research and development of online learning systems for several years, key areas of interest being the design of learning environments and use of online activities. Recent research includes the use of educational games with adult learners. Dr John Ford is a Senior Lecturer in the Institute of Petroleum Engineering at Heriot-Watt University. His research focuses on the area of Drilling Engineering but he has also initiated studies on the use of novel techniques in the delivery of Masters-level and professional development courses. Dr Nils Tomes is Director e-Networks and Communities for the British Council. She was Director of Learning Technology at Heriot-Watt University and developed a national online programme for post-compulsory education. This programme is now in use by science, maths and language pupils within all education authorities in Scotland and is being adopted in England, Southeast Asia and Southern Asia. 